\title{
Solving the Pitfalls of Bimanual Phacoemulsification - Oval Instruments Do Save Energy
}

a report by

\author{
Fernando Araújo-Gomes
}

Ophthalmology Surgeon, Clínica de Santo António

DOI: 10.17925/EOR.2007.00.00.39

In physics, energy transfer per unit of time equals power, or the amount of work done. The use of fluid as a tool to carry out work is known as hydraulics. In hydraulics, the use of the kinetic energy of a moving fluid is known as hydrodynamics, and the use of the static pressure of a fluid contained in a closed system with a reservoir and piping is known as hydrostatics.

\section{Hydrodynamics and Hydrostatics}

Phacoemulsification should be regarded as a complex system comprising a hydraulic machine with piping, providing work or power to a chamber - the eye. The work inside this chamber becomes a hydrodynamic process with fluid flow and pressure depending on the phaco machine itself. The whole system - made up of the machine and the eye - constitutes a hydrostatic power transmission system, with a reservoir for the fluid and tubing and a pump to drive the fluid. The difference lies in the pump - a vacuum pump, which is needed to provide the fluidics in the anterior chamber - and in the fact that the fluid is not re-used. Basically, however, the system uses the power transmission of the static pressure that stands inside the tubing.

From any handbook of physics we can learn that, since the connection between the elements is through the piping, the principal advantage of hydrostatic power transmission (see Figure 1) is that these elements can be positioned in whatever fashion is suitable for the machine manufacturer. Hydrostatic transmission also has the advantage of not depending on the size of components.

However, the main disadvantage of hydrostatic drive is that it may lose efficiency due to internal leakage, fluid-flow losses or mechanical friction losses (see Figure 2), all of which result in power being wasted. In addition, if for any reason any external leakage or fluid loss is apparent, more power is wasted and the system becomes more inefficient. Assuming that we are dealing with hydrostatic transmission, our aim in terms of phacoemulsification itself is to spare the energy, or power, lost through leakage. What happens, then, if our phacoemulsification is made bimanually with a sleeveless round needle?

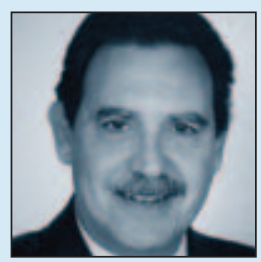

Fernando Araújo-Gomes is an ophthalmology surgeon in private practice at Clínica de Santo António, Amadora, Portugal, and in office-based practice in Lisbon, Portugal. He is a member of the Portuguese Medical Association, the Portuguese Society of Ophthalmology and the European Society of Cataract and Refractive Surgeons (ESCRS). Dr Araújo-Gomes has lectured internationally and has presented several instructional courses on cataracts and multifocal intraocular lenses. He developed his own technique and instrumentation for bimanual ultra-smallincision cataract surgery.
Incisions - Size, Shape, Misconceptions and

Frequent Mistakes

It is important, before going on to leakage, to understand common mistakes and misunderstandings surrounding bimanual phacoemulsification.

\section{Size}

Most irrigating choppers currently in use are 19G. Until now, irrigating choppers and phaco needles have been round. This has meant the introduction into the elastic tissue of the limbus of a circular instrument with a certain gauge through a linear incision. Since it is very common to read or hear of surgery performed using a $1 \mathrm{~mm}$ incision, we will take this measurement as our example. We will consider a $19 \mathrm{G}$ instrument with a circular section of $1 \mathrm{~mm}$ in outer diameter. It is this circle that needs to be introduced through a $1 \mathrm{~mm}$ incision. Using the formula $2 \pi r$, for a diameter of $1 \mathrm{~mm}$ we calculate a perimeter of $3.1416 \mathrm{~mm}$. As the limbus is vertically elastic, the incision will open equally up and down, making the size of the incision half of the perimeter just $1.57 \mathrm{~mm}$. This means that for a $19 \mathrm{G}$ instrument (1 $\mathrm{mm}$ in diameter) the real size of the incision needed would be $1.57 \mathrm{~mm}$. The same considerations work for a $20 \mathrm{G} 0.9 \mathrm{~mm}$ instrument, which would require an opening of $1.41 \mathrm{~mm}$. This is a lot greater than $1 \mathrm{~mm}$; therefore, it is probable that when we hear or read about $1 \mathrm{~mm}$ incisions, the author is actually referring to $1 \mathrm{~mm}$ instruments. These disparities are due to the fact that the diameter of the instruments is reported to be equal to the size of the incisions. This in itself is based on the assumption that the incision fits perfectly around the circular instruments. This is the second mistake - as well as the size of the incision being misjudged, the shape of the incision is unclear.

\section{Shape}

As previously noted, conventional phaco instruments are round, meaning their section is a circle. In a circle the diameter is constant at any point. However, we must understand that in phaco surgery we are dealing with the perimeter of the incision, not with the diameter of the instrument.

A knife produces a linear opening, and the elasticity in the limbus or cornea does not allow us to transform this line into a circle, even if the incision is forced. Forcing it would make the surgical incision into a traumatic wound, which would be out of the question. The lack of elasticity in the vertical plane of the limbus means that the incision must be widened horizontally to allow the smooth entrance of the round instrument without lacerating. The best achievable result, therefore, using a gentle and precise technique, is an oval opening. The incision, therefore, does not match the circular shape of the instrument. When we consider the size of the incision we must envisage an oval and forget the circle, which is the source of all misunderstandings. Furthermore, instead of one single diameter, we now have one minor vertical axis and one major horizontal axis. In other words, if vertical elasticity allows the 


\section{Cataracts}

Figure 1: A Basic Hydraulic Power Transmission System

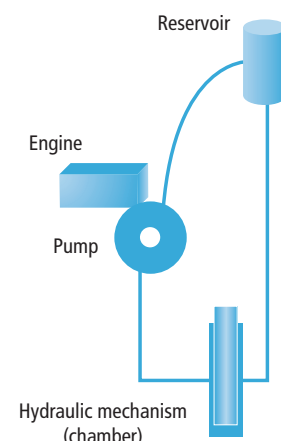
(chamber)

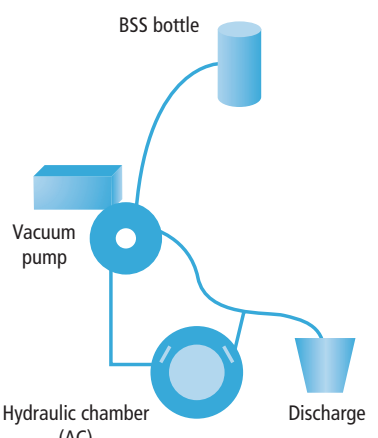

(AC)
Figure 2: Excessive Fluid Loss

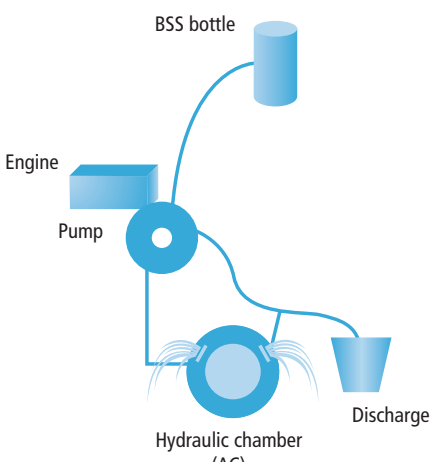

(AC)

Figure 3: Conventional Phaco with Silicone - No Leakage

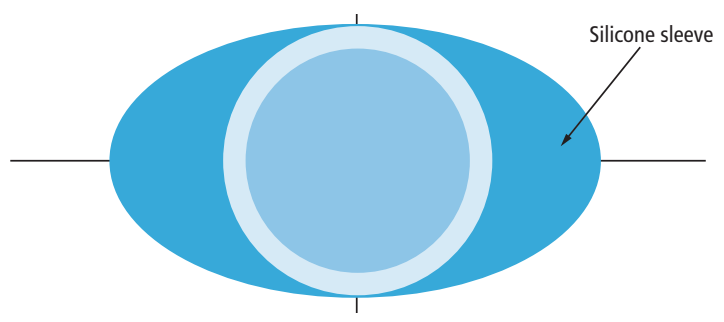

Figure 4: Bimanual Phaco without Silicone Sleeve, Round - Leakage

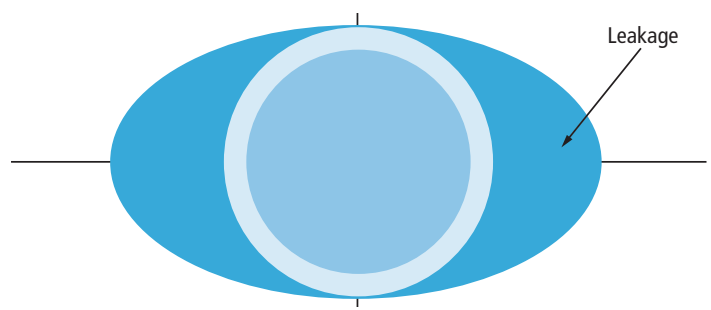

Figure 5: Bimanual Phaco without Silicone Sleeve, Oval Minimal Leakage

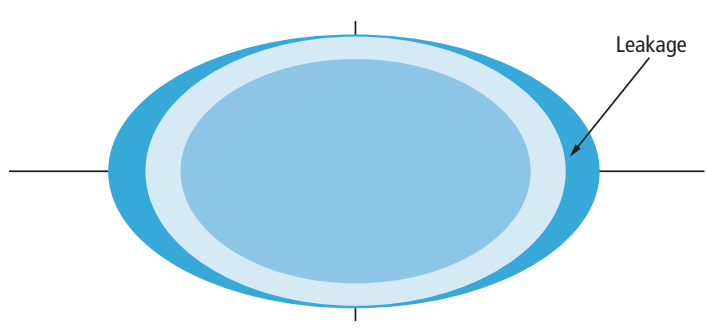

entrance of the diameter of a round instrument, this will be the minor axis of the oval opening; horizontally, due to the lack of elasticity, we must widen the incision until a forceless entrance is possible - this will be the larger or major axis of the oval opening, and approximately the real size of the incision. When a round instrument is used, since the diameter is constant, it is in this horizontal plane that a space for leakage is created. However, as with the diameter of the circle, what we must consider for sizing the incision is not the size of the major axis or the minor axis, but half the perimeter of the oval (see Figures 3-6).

The True Shape and the Real Incision Size

It is concluded, therefore, that with round instruments (choppers and phaco needles) a circle is introduced into an oval opening. With conventional coaxial phaco, however, the needle is surrounded by a silicone sleeve, and this is the real difference between the techniques. This silicone sleeve matches the shape of the incision, while the bare instrument does not, as explained above. With the sleeve, therefore, excessive leakage does not occur. Furthermore, the second hand holds a thin conventional chopper through an opening smaller than $1 \mathrm{~mm}$, with leakage leaving the system through the 'escape valve' that every closed system needs. However, removing the sleeve from the phaco needle and using a round irrigating chopper in the second hand reveals space around the instrument, allowing excess leakage. The second hand, which holds the irrigating chopper, experiences the same problem. This creates a level of leakage that is dangerous and compromises the system. Bearing in mind the flow losses in the hydrostatic system, this is the main reason why chambers collapse.

This raises doubts about the benefits of using the bimanual technique. Bimanual, however, has several advantages over coaxial. First, the bimanual technique separates irrigation from aspiration, which is why it is referred to as biaxial surgery. The separate irrigating axis, in the second hand, provides the surgeon with another tool. By freely directing the fluid in several directions during the procedure, the surgeon can move the nuclear fragments from the angle to the aspirating tip, use the flow as a holder for the posterior capsule and even stabilise a floppy iris. However, directing the fluid is possible only if the irrigating chopper has a frontal, open-ended irrigation. Bimanual also enables the use of a truly ultra-small incision. Despite new evolutions in conventional phaco tips, I believe that only a sleeveless needle provides a truly small incision $-1.4 \mathrm{~mm}$ or less.

Finally, bimanual offers advantages in terms of fluidics. As we are working with ultra-small incisions, if we solve the leakage problem, we will achieve a much more efficient relationship of inflow to outflow in the anterior chamber, which will result in a safer and more predictable surgery. This is the key point on which everything else depends.

It is because of these advantages that we must go on trying to solve the leakage problem.

The True Shape - Saving Energy by Reducing Leakage Surgeons trying to compensate for this excessive leakage tend to raise the fluid bottle levels to around $1 \mathrm{~m}$, slow the pump velocity, lower the aspirating settings or use higher sizes for the chopper in order to achieve more flow. External pumping systems are also occasionally used in order to make the inflow more efficient. In spite of all these adaptations, the anterior chamber is barely stabilised and is placed under too much stress. So far, no advantages are evident over the coaxial technique, which is frustrating.

To summarise, we hope to preserve the advantages of an ultra-small incision and the benefits of separate irrigation and aspiration. However, with a smaller incision the sleeve must be removed, and in doing this too 
much leakage is evoked, causing the chamber to collapse. The problem of excessive leakage depends on the shape of the instruments, and the gain in efficiency on the size of the incision. The only way to solve the shape conflict, if the incision is definitely oval, is to use oval instruments.

The True Size - Final Calculations on the Oval Shape The following formula can be used to calculate the perimeter or circumference of an oval shape:

$$
20 \sqrt{1 / 2\left(a^{2}+B^{2}\right)} .
$$

Take the example of a $20 \mathrm{G}$ implement (see Figure 6). The area of a $20 \mathrm{G}$ circle is 0.5 . To achieve a 0.5 oval, given $1 \mathrm{~mm}$ as the major axis, and using the above formula, the minor axis must be $0.6 \mathrm{~mm}$. Taking $1 \mathrm{~mm}$ and $0.6 \mathrm{~mm}$ as the axes, the perimeter will be $2.65 \mathrm{~mm}$. The incision should therefore be half the perimeter $-1.32 \mathrm{~mm}$. Note that, even with a $1 \mathrm{~mm}$ major axis, the incision will never be $1 \mathrm{~mm}$. Ultra-small-incision cataract surgery (USICS) is my personal designation for an effective bimanual cataract surgery using an incision no greater than $1.5 \mathrm{~mm}$. Incisions under $1.5 \mathrm{~mm}$ with minor leakage, and a well-fitting instrument with an oval opening, produce better fluidics inside the anterior chamber, hence a more stable and effective surgery. Using the half-perimeter of the instrument as the real size of the incision, and using oval shapes to provide a better fitting, are the key issues to resolve the pitfalls of bimanual sleeveless phaco surgery.

\section{The A Gomes Ultra-small-incision Cataract Surgery Kit}

Based on these conclusions, I designed an oval irrigating chopper and, most importantly, an oval needle; without this oval needle, the whole technique would be ineffective. I selected $1.2 \mathrm{~mm}$ and $0.6 \mathrm{~mm}$ as the axes for the oval instruments, with $20 \mathrm{G}$ able to pass through $1.4 \mathrm{~mm}$, in order to retain the concept of a surgery with an incision smaller than $1.5 \mathrm{~mm}$. Buerki Innomed, in Switzerland, manufactured these instruments and they have since been put to use on a regular basis. As well as the chopper and needle, there is one irrigation handpiece and one aspiration handpiece, which are both oval. The set was named the A Gomes USICS Kit. After more than 750 consecutive cataracts, ranging from + to ++++, the oval instruments have proved to work perfectly. They have been used with Pentasys, Millennium and Sovereign machines, without any of the regular settings needing to be changed, and without the occurence of any flat chambers. They work with pump velocities of up to $40 \mathrm{cc} / \mathrm{mn}$, without the bottle increasing to more than $75 \mathrm{~cm}$ (25 inches) high.

Since I consider bimanual surgery to be a technique involving incisions of less than $1.5 \mathrm{~mm}$, and as we now have some intraocular lenses (IOLs) that go through incisions of $1.75 \mathrm{~mm}$, I assume that with these instruments we can perform the whole surgery, from beginning to end, with an incision of no greater than $1.8 \mathrm{~mm}$. This surpasses the currently accepted standards, which define bimanual surgery as surgery with incisions under $2 \mathrm{~mm}$. For softer cataracts or for almost clear lenses, as for presbyopic lens exchange, I am now using the same concept but with $21 \mathrm{G}$ instruments, which work through $1.2 \mathrm{~mm}$ incisions.

\section{Conclusion}

Bimanual cataract surgery has many advantages over conventional phaco surgery. It provides the surgeon with an independent irrigation line, allowing biaxial surgery. It allows the benefits of a truly small incision. Once oval instruments are put to use it will mean a better relationship of
Figure 6: 20G Instruments

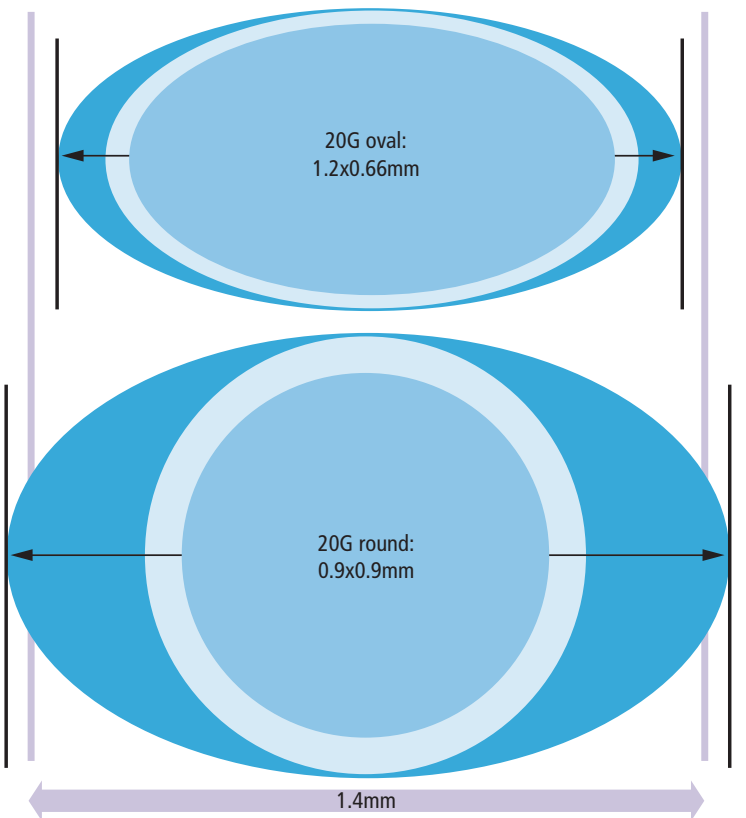

Note differences in incision size and leakage.

inflow to outflow. Altogether, these elements form a more closed system, which enables a very safe and predictable surgery. The use of this small-incision technique means the surgeon can insert the latest developments in small-incision IOLs.

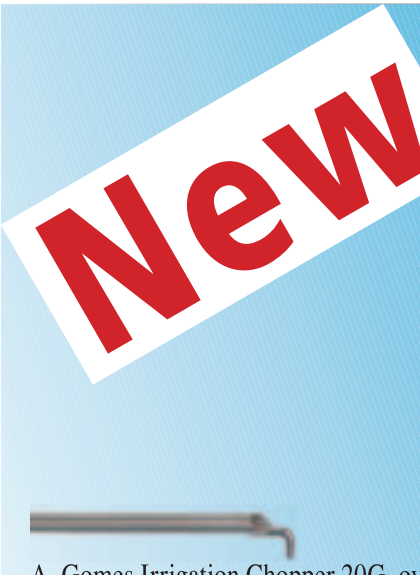

A. Gomes Irrigation Chopper 20G, oval cannula with nucleus manipulator and with frontal irrigation

A. Gomes Aspiration handpiece 21G, oval cannula with rough tip and oval front aspiration port

\section{Product Features}

- Ideal kit for bimanually USICS (ultra small incisions cataract surgery)

- Elliptical shape to provide a better fitting to the incision, hence less leakage and better Anterior Chamber stability

- Better visualization of the Chopper hook

Ergonomically designed handpieces with angulations developed to provide the surgeon stress less hand positions

Works through $1.4 \mathrm{~mm}$ or less incision 